TRANSACTIONS OF THE

AMERICAN MATHEMATICAL SOCIETY

Volume 355, Number 5 , Pages 2047-2064

S 0002-9947(02)03232-4

Article electronically published on December 18, 2002

\title{
NON-SOLVABILITY FOR A CLASS OF LEFT-INVARIANT SECOND-ORDER DIFFERENTIAL OPERATORS ON THE HEISENBERG GROUP
}

\author{
DETLEF MÜLLER AND MARCO M. PELOSO
}

\begin{abstract}
We study the question of local solvability for second-order, leftinvariant differential operators on the Heisenberg group $\mathbb{H}_{n}$, of the form

$$
\mathcal{P}_{\Lambda}=\sum_{i, j=1}^{n} \lambda_{i j} X_{i} Y_{j}={ }^{t} X \Lambda Y,
$$

where $\Lambda=\left(\lambda_{i j}\right)$ is a complex $n \times n$ matrix. Such operators never satisfy a cone condition in the sense of Sjöstrand and Hörmander. We may assume that $\mathcal{P}_{\Lambda}$ cannot be viewed as a differential operator on a lower-dimensional Heisenberg group. Under the mild condition that $\operatorname{Re} \Lambda, \operatorname{Im} \Lambda$ and their commutator are linearly independent, we show that $\mathcal{P}_{\Lambda}$ is not locally solvable, even in the presence of lower-order terms, provided that $n \geq 7$. In the case $n=3$ we show that there are some operators of the form described above that are locally solvable. This result extends to the Heisenberg group $\mathbb{H}_{3}$ a phenomenon first observed by Karadzhov and Müller in the case of $\mathbb{H}_{2}$. It is interesting to notice that the analysis of the exceptional operators for the case $n=3$ turns out to be more elementary than in the case $n=2$. When $3 \leq n \leq 6$ the analysis of these operators seems to become quite complex, from a technical point of view, and it remains open at this time.
\end{abstract}

\section{INTRODUCTION}

While the question of local solvability for principal type operators has been solved in a series of fundamental papers $([\mathrm{L},[\mathrm{H1}],[\mathrm{H} 2,[\mathrm{G},[\mathrm{NT},[\mathrm{BF}$, just to name a few), the same question for multiply characteristic partial differential operators with smooth coefficients is still a wide open problem. Some partial results appear for instance in [H3], [BGH], [S] in the general case, and in [HeN], L-B], [CR], [R], [MR1], [MR2], [MR3], [MPR] in the setting of translation invariant differential operators on a nilpotent Lie group.

In this paper we restrict our attention to the case of the Heisenberg group $\mathbb{H}_{n}$ and analyze a particular class of second-order, left-invariant differential operators on $\mathbb{H}_{n}$ which are doubly characteristic. This class consists of the operators of the

Received by the editors October 8, 2002.

2000 Mathematics Subject Classification. Primary 35A05, 35D05, 43A80.

Key words and phrases. Local solvability, Heisenberg group.

We acknowledge the support for this work by the European Commission through the European TMR network "Harmonic Analysis" and the IHP Network HARP "Harmonic Analysis and Related Problems". 
form

$$
\mathcal{P}_{\Lambda}=\sum_{i, j=1}^{n} \lambda_{i j} X_{i} Y_{j}={ }^{t} X \Lambda Y
$$

where $X_{j}=\partial_{x_{j}}-\frac{1}{2} y_{j} \partial_{t}, Y_{j}=\partial_{y_{j}}+\frac{1}{2} x_{j} \partial_{t},(x, y, t) \in \mathbb{R}^{n} \times \mathbb{R}^{n} \times \mathbb{R}$ denote the coordinates in $\mathbb{H}_{n}$ and $\Lambda=\left(\lambda_{i j}\right)$ is an $n \times n$ complex matrix. Notice that these vector fields satisfy the Heisenberg commutation relations $\left[X_{i}, X_{j}\right]=\left[Y_{i}, Y_{j}\right]=$ $0,\left[X_{i}, Y_{j}\right]=\delta_{i, j} T, i, j=1, \ldots, n$, where $T=\partial_{t}$. To avoid trivialities, we shall always assume that $n \geq 2$. We remark that local solvability for second-order leftinvariant differential operators on the lowest-dimensional Heisenberg group $\mathbb{H}_{1}$ is by now completely understood, see [MPR], [MR1], [MZ].

Particular operators of the form (10) first appeared (in disguised form) in [MPR in the lowest-dimensional case of interest, $\mathbb{H}_{2}$. This case was later analyzed in [KM], and it showed some peculiar new features in the theory of local solvability for translation-invariant operators on nilpotent Lie groups. More precisely, in the case when $n=2$, G. Karadzhov and the first named author characterized the locally solvable operators $\mathcal{P}_{\Lambda}$ for $\Lambda$ in a Zariski open subset. In particular, they showed that $\mathcal{P}_{\Lambda}$ can be locally solvable even if $\mathcal{P}_{\Lambda}$ does not satisfy a cone condition. We say that an operator $\mathcal{P}_{\Lambda}$ satisfies a cone condition at a point $\left(x_{0}, y_{0}, t_{0}\right)$ if the image of its principal symbol at this point is contained in a proper cone in the complex plane. Since we are dealing with (left-) invariant differential operators, it suffices to analyze local solvability at the origin in $\mathbb{H}_{n}$.

In the recent paper [MR3, F. Ricci and D. Müller showed that if $\mathcal{L}$ is a secondorder, left-invariant differential operator on the Heisenberg group $\mathbb{H}_{n}$ satisfying a cone condition, then, generically, $\mathcal{L}$ is solvable.

In this paper we continue the analysis begun in $[\mathrm{KM}$ and study the operators of the form (1) for general $n \geq 2$.

Let us say that $\mathcal{P}_{\Lambda}$ is reducible to the Heisenberg group $\mathbb{H}_{m}$ (with $m \leq n$ ) if it can be written in the form $\mathcal{P}_{\Lambda}=\sum_{i, j=1}^{m} \tilde{\lambda}_{i j} \tilde{X}_{i} \tilde{Y}_{j}$, where the $\tilde{\lambda}_{i j}$ are complex coefficients and the left-invariant vector fields $\tilde{X}_{j}, \tilde{Y}_{j}$ satisfy the Heisenberg commutation relations $\left[\tilde{X}_{i}, \tilde{X}_{j}\right]=\left[\tilde{Y}_{i}, \tilde{Y}_{j}\right]=0,\left[\tilde{X}_{i}, \tilde{Y}_{j}\right]=\delta_{i, j} T, i, j=1, \ldots, m$. Clearly, $\mathcal{P}_{\Lambda}$ can then be regarded as a differential operator on the Heisenberg group $\mathbb{H}_{m}$. We denote by $n\left(\mathcal{P}_{\Lambda}\right)$ the minimal $m$ such that $\mathcal{P}_{\Lambda}$ can be reduced to the Heisenberg group $\mathbb{H}_{m}$. We shall show that if $n\left(\mathcal{P}_{\Lambda}\right) \geq 7$ and if the matrices $\operatorname{Re} \Lambda, \operatorname{Im} \Lambda$ and $[\operatorname{Re} \Lambda, \operatorname{Im} \Lambda]$ are linearly independent, then the operator $\mathcal{P}_{\Lambda}+\mathcal{L}$ is not locally solvable, for every first-order differential operator $\mathcal{L}$ with smooth coefficients on $\mathbb{H}_{n}$.

It is known $\overline{\mathrm{KM}}$ that when $n\left(\mathcal{P}_{\Lambda}\right)=2$ there are some operators, which we call exceptional, that do not satisfy the cone condition and that are in fact locally solvable. In this paper we show that this phenomenon also occurs when $n\left(\mathcal{P}_{\Lambda}\right)=3$, see Proposition [1.2 The complete analysis of the remaining cases $3 \leq n\left(\mathcal{P}_{\Lambda}\right) \leq$ 6 seems to become quite complex from a technical point of view (even though presumably not very enlightening), and it remains open at this time. Our example in Proposition 1.2 indicates that there may exist further exceptional operators for $n(\mathcal{P})$ in this range. 


\section{Statement of the main Results \\ AND SOME AUXILIARY FACTS}

Let $\Lambda$ be a complex $n \times n$ matrix, $\Lambda=A+i B$ with $A$ and $B$ real, and let $C=[A, B]$. We say that Hörmander's condition $(\mathrm{H})$ holds if:

(H) There exists $(\xi, \eta) \in \mathbb{R}^{2 n}$ such that

$$
{ }^{t} \xi A \eta={ }^{t} \xi B \eta=0 \quad \text { and } \quad{ }^{t} \xi C \eta \neq 0 .
$$

Remarks. It is an easy consequence of Hörmander's general criterium for local non-solvability in $\left[\mathrm{H} 2\right.$ that $\mathcal{P}_{\Lambda}+\mathcal{L}$ is nowhere locally solvable, for any first-order differential operator with smooth coefficients if condition $(\mathrm{H})$ is satisfied.

Moreover, if $A$ and $B$ are linearly dependent, then, after multiplying $\mathcal{P}_{\Lambda}$ by a suitable complex scalar, we may assume that $B=0$. Since the case of operators with real coefficients is covered by [MR2], we may assume that $A$ and $B$ are linearly independent.

We shall in fact assume throughout the paper that the following, stronger condition (I) holds, which obviously is a necessary condition in order for $(\mathrm{H})$ to hold.

(I) The matrices $A, B$ and $C$ are linearly independent.

We can now state our main result.

Theorem 1.1. Let $\mathcal{P}=\mathcal{P}_{\Lambda}$ and $\Lambda$ be as above and let $\mathcal{L}$ denote any first-order differential operator with $C^{\infty}$ coefficients (not necessarily invariant). Let $n(\mathcal{P}) \geq 7$ and suppose condition (I) holds. Then also condition (H) holds, so that $\mathcal{P}+\mathcal{L}$ is not locally solvable.

When $n(\mathcal{P})=3$, the situation is no longer so plain, as the following example indicates.

Proposition 1.2. On $\mathbb{H}_{3}$, consider the operators of the form

$$
\mathcal{P}=(1+i \alpha) X_{1} Y_{2}+i \beta X_{1} Y_{3}+i \gamma X_{2} Y_{2}+(1+i \delta) X_{2} Y_{3},
$$

where $\alpha, \beta, \gamma, \delta \in \mathbb{R}$.

Assume that condition (I) holds. Then condition (H) holds if and only if $\gamma=0$ or $\gamma \neq 0$ and $(\delta-\alpha)^{2}+4 \beta \gamma>0$; hence $\mathcal{P}+\mathcal{L}$ is not locally solvable, for any first-order differential operator $\mathcal{L}$ with $C^{\infty}$ coefficients, under these conditions.

If $\gamma \neq 0$ and $(\delta-\alpha)^{2}+4 \beta \gamma \leq 0$, then the operator $\mathcal{P}$ admits a tempered fundamental solution and hence is locally solvable.

Let us remark that, as we shall show in Section 3, the operators (2) arise in a natural way if we assume that $A$ has Jordan normal form given by

$$
A=\left(\begin{array}{lll}
0 & 1 & 0 \\
0 & 0 & 1 \\
0 & 0 & 0
\end{array}\right) .
$$

Moreover, the subsequent discussion in Sections $3-5$ indicates that a complete study of local solvability of the operators $\mathcal{P}_{\Lambda}$ in the cases where $3 \leq n\left(\mathcal{P}_{\Lambda}\right) \leq 6$ would presumably require a technically complex but not very exciting case-by-case study of a great number of different situations, corresponding to all possible normal forms. This is why we restrict ourselves here to the study of the class of operators (2).

The condition $A, B$ linearly independent has a simple geometric interpretation, as the following proposition shows. We denote by $\sigma_{L}$ the principal symbol of a 
differential operator $L$. Observe that the principal symbol of $\mathcal{P}_{\Lambda}$ at the origin is given by $\sigma_{\mathcal{P}_{\Lambda}}(0,(\xi, \eta, \tau))=-{ }^{t} \xi \Lambda \eta$.

Proposition 1.3. Let $\mathcal{P}=\mathcal{P}_{\Lambda}$ be as in (1). Then the range of $\sigma_{\mathcal{P}}$ is always an $\mathbb{R}$-linear subspace of the complex plane, and range $\sigma_{\mathcal{P}}=\mathbb{C}$ if and only if $A$ and $B$ are linearly independent. In particular, if $\Lambda \neq 0$, then $\mathcal{P}$ never satisfies a cone condition.

Proof. Suppose that $A$ and $B$ are linearly independent. Assume first that there exists an $x \in \mathbb{R}^{n}$ such that $A x \wedge B x \neq 0$. Then the mapping

$$
y \mapsto\left(\begin{array}{l}
y \cdot A x \\
y \cdot B x
\end{array}\right)
$$

is onto, i.e., range $\sigma_{\mathcal{P}}=\mathbb{C}$. Suppose next that $A x \wedge B x=0$ for all $x$. Then the discussion of Case 1(a) in the proof of Theorem 1.1 will show that necessarily $\operatorname{rank} B<2$ and $\operatorname{rank} A<2$, since $A$ and $B$ are linearly independent. Then $\operatorname{rank} A=$ $\operatorname{rank} B=1$, so that

$$
A x=\left(x \cdot \xi_{1}\right) \eta, \quad B x=\left(x \cdot \xi_{2}\right) \eta,
$$

with $\xi_{1}, \xi_{2}$ linearly independent (compare the discussion of Case 1(b) in the proof of Theorem 1.11). Then

$$
\begin{aligned}
\sigma_{\mathcal{P}}(0,(x, y)) & ={ }^{t} x A y+i{ }^{t} x B y \\
& =\left(y \cdot \xi_{1}\right)(x \cdot \eta)+i\left(y \cdot \xi_{2}\right)(x \cdot \eta) \\
& =(x \cdot \eta) y \cdot\left(\xi_{1}+i \xi_{2}\right) ;
\end{aligned}
$$

i.e., $\sigma_{\mathcal{P}}$ has range $=\mathbb{C}$. The case where $A$ and $B$ are linearly dependent is obvious.

The following sufficient condition for $\mathcal{P}_{\Lambda}$ to be reducible to a lower-dimensional Heisenberg group will be useful.

Proposition 1.4. Let $\Lambda=A+i B$ and $\mathcal{P}=\mathcal{P}_{\Lambda}$ be as above. Let

$$
V=\operatorname{range} A+\operatorname{range} B, \quad V^{\prime}=\operatorname{coker} A+\operatorname{coker} B .
$$

Then $V+V^{\prime}$ is invariant under $A$ and $B$, and $\mathcal{P}$ is reducible to a lower-dimensional Heisenberg group, provided $V+V^{\prime} \neq \mathbb{R}^{n}$.

Proof. Notice that the inner product on $\mathbb{R}^{n}$ is fixed here as the canonical inner product, in view of Hörmander's condition. Then coker $A$ and coker $B$ are well defined by the equality coker $A=\operatorname{range}{ }^{t} A=(\operatorname{ker} A)^{\perp}$ (and similarly for $B$ ), and $V+V^{\prime}$ is invariant under $A$ and $B$.

Hence, $\mathbb{R}^{n}=\left(V+V^{\prime}\right) \oplus K$, where $K \subset \operatorname{ker} A \cap \operatorname{ker} B$. Choose a basis $\tilde{e}_{1}, \ldots, \tilde{e}_{n}$ of $\mathbb{R}^{n}$ such that $V+V^{\prime}=\operatorname{span}\left\{\tilde{e}_{1}, \ldots, \tilde{e}_{m}\right\}, K=\operatorname{span}\left\{\tilde{e}_{m+1}, \ldots, \tilde{e}_{n}\right\}$, and write $e_{i}=\sum_{k=1}^{n} t_{i k} \tilde{e}_{k}, i=1, \ldots, n$, where $e_{1}, \ldots, e_{n}$ denotes the canonical basis of $\mathbb{R}^{n}$. Let $T=\left(t_{i j}\right)$, write $T^{-1}=\left(t^{i j}\right)$ and put

$$
\tilde{X}_{i}=\sum_{k} t^{i k} X_{k}, \quad \tilde{Y}_{j}=\sum_{l} t_{l j} Y_{l} .
$$

Then $\mathcal{P}=\sum_{i, j} \lambda_{i j} X_{i} Y_{j}=\sum_{k l} \tilde{\lambda}_{k l} \tilde{X}_{k} \tilde{Y}_{l}$, where $\tilde{\lambda}_{k l}=\sum_{i, j} \lambda_{i j} t_{i k} t^{l j}$, i.e., $\tilde{\Lambda}=\left(\tilde{\lambda}_{k, l}\right)$ is given by $\tilde{\Lambda}={ }^{t} T \Lambda^{t} T^{-1}$. Moreover, one checks immediately that the vector fields $\tilde{X}_{1}, \ldots, \tilde{X}_{n}, \tilde{Y}_{1}, \ldots, \tilde{Y}_{n}$ satisfy the Heisenberg commutation relations. Also, if $k>$ 
$m$, then we have $\tilde{e}_{k} \in K \subset \operatorname{ker} \Lambda$ and $\tilde{e}_{k}=\sum_{i} t^{k i} e_{i}={ }^{t} T^{-1} e_{k}$, so that $\tilde{\Lambda} e_{k}=0$. Similarly, since range $\Lambda \subset \operatorname{span}_{\mathbb{C}}\left\{\tilde{e}_{1}, \ldots, \tilde{e}_{m}\right\}$, one has range $\tilde{\Lambda} \subset \operatorname{span}_{\mathbb{C}}\left\{e_{1}, \ldots, e_{m}\right\}$, so that $\tilde{\lambda}_{k, l}=0$, unless $k \leq m$ and $l \leq m$. This shows that $\mathcal{P}$ is reducible to $\mathbb{H}_{m}$, where $m<n$, if $V+V^{\prime} \neq \mathbb{R}^{n}$.

\section{Proof of Theorem 1.1}

The proof of Theorem 1.1 is somewhat lengthy and is divided into several cases, according to the following scheme:

Case 1. $A x \wedge B x=0$ for all $x \in \mathbb{R}^{n}$ :

- Subcase (a) $\operatorname{rank} A \geq 2$ or $\operatorname{rank} B \geq 2$;

- Subcase (b) $\operatorname{rank} A=\operatorname{rank} B=1$.

Case 2. $A x \wedge B x \neq 0$ for some $x \in \mathbb{R}^{n}$.

- Subcase (a) $A x \wedge A y \wedge B x \wedge B y \neq 0$ for some $x, y \in \mathbb{R}^{n}$;

- Subcase (b) dim $\operatorname{span}\{A x, A y, B x, B y\} \geq 3$ for some $x, y \in \mathbb{R}^{n}$, but (a) does not hold;

- Subcase (c) dim $\operatorname{span}\{A x, A y, B x, B y\} \leq 2$ for all $x, y \in \mathbb{R}^{n}$.

We shall make the general assumption that condition (I) holds, and remark that the condition $n\left(\mathcal{P}_{\Lambda}\right) \geq 7$ will only be relevant in Subcases 2 (b) and 2 (c).

Case 1(a). Suppose e.g. that $\operatorname{rank} B \geq 2$. By assumption, for every $x$ with $B x \neq 0$ there exists a unique $\alpha(x) \in \mathbb{R}$ such that

$$
A x=\alpha(x) B x .
$$

Since rank $B \geq 2$, there are $x_{0}, y_{0}$ such that $B x_{0} \wedge B y_{0} \neq 0$, and hence the set $\left\{(x, y) \in \mathbb{R}^{n} \times \mathbb{R}^{n}: B x \wedge B y \neq 0\right\}$ is Zariski open in $\mathbb{R}^{2 n}$.

In the sequel, we shall say that a property holds generically if it holds for all points in a nonempty, Zariski open subset. Thus, for generic $(x, y)$, we have $B(x+y) \neq 0$, and

$$
\left\{\begin{array}{l}
A(x+y)=\alpha(x+y)(B x+B y) \\
A(x+y)=A x+A y=\alpha(x) B x+\alpha(y) B y .
\end{array}\right.
$$

Since $B x$ and $B y$ are generically linearly independent, it follows that, generically,

$$
\alpha(x+y)=\alpha(x) \quad \text { and } \quad \alpha(x+y)=\alpha(y) .
$$

Let $\left\{v_{1}, \ldots, v_{n}\right\}$ be a basis of $\mathbb{R}^{n}$ such that these equations hold for some $x=x_{0}$ and $y$ from this basis. Then

$$
\alpha\left(v_{j}\right)=\alpha\left(x_{0}+v_{j}\right)=\alpha\left(x_{0}\right)=: \alpha_{0} .
$$

Hence, $A v_{j}=\alpha_{0} B v_{j}$ for $j=1, \ldots, n$, i.e. $A=\alpha_{0} B$.

Consequently, under the hypothesis (I), Subcase 1(a) cannot arise.

Case 1(b). If $\operatorname{rank} A=\operatorname{rank} B=1$, then there are vectors $\xi_{j}, \eta_{j}, j=1,2$, such that $A x=\left(x \cdot \xi_{1}\right) \eta_{1}$ and $B x=\left(x \cdot \xi_{2}\right) \eta_{2}$. Since $A x \wedge B x=0$ for all $x, \eta_{1}=c \eta_{2}$. After rescaling, we may assume that

$$
A x=\left(x \cdot \xi_{1}\right) \eta, \quad B x=\left(x \cdot \xi_{2}\right) \eta .
$$

Clearly, range $C \subseteq \operatorname{span}\{\eta\}$, so that there exists $\xi_{3}$ such that

$$
C x=\left(x \cdot \xi_{3}\right) \eta \text {. }
$$


Since (I) holds, $\xi_{3} \notin \operatorname{span}\left\{\xi_{1}, \xi_{2}\right\}$. Let $y \perp \operatorname{span}\left\{\xi_{1}, \xi_{2}\right\}$, but $y \not \perp \xi_{3}$. Then

$$
{ }^{t} x A y={ }^{t} x B y=0 \quad \text { for all } x, \quad \text { and } \quad C y \neq 0 .
$$

This shows that $(\mathrm{H})$ holds (and necessarily $n \geq 3$ ).

Henceforth, we shall always assume that $n \geq 3$ (since the case $n=2$ is covered by $[\mathrm{KM}]$ ), and that $(\mathrm{H})$ fails to hold. Moreover, since we shall disprove local solvability in Theorem 1.1 always by means of Hörmander's criterion, which is a condition on the principal symbol only, we can and will assume without loss of generality that $n\left(\mathcal{P}_{\Lambda}\right)=n$. In most cases, our assumptions will lead to a contradiction, so that in fact $(\mathrm{H})$ must be true.

Case 2. Let $U=\{x: A x \wedge B x \neq 0\}$. Then $U$ is a conic, Zariski-open set in $\mathbb{R}^{n}$. Then, for all $x \in U$ there exist unique $\alpha(x), \beta(x) \in \mathbb{R}$ such that

$$
C x=\alpha(x) A x+\beta(x) B x .
$$

Notice that $\alpha, \beta$ are rational functions, more precisely, quotients of quadratic forms, homogeneous of degree 0 , and that $\alpha, \beta$ depend analytically on the coefficients of $A$ and $B$, since $\alpha(x) A x \wedge B x=C x \wedge B x$ and $\beta(x) B x \wedge A x=C x \wedge A x$.

For generic $x, y \in U$, also $x+y$ lies in $U$, so that

$$
\begin{aligned}
C(x+y) & =\alpha(x+y) A(x+y)+\beta(x+y) B(x+y) \\
& =\alpha(x+y)(A x+A y)+\beta(x+y)(B x+B y),
\end{aligned}
$$

but also

$$
\begin{aligned}
C(x+y) & =C x+C y \\
& =\alpha(x) A x+\alpha(y) A y+\beta(x) B x+\beta(y) B y .
\end{aligned}
$$

Subcase 2(a). We assume that dim $\operatorname{span}\{A x, A y, B x, B y\}=4$ for some $(x, y)$, hence also for generic $(x, y)$. From equations (4) and (5) it follows that, generically,

$$
\begin{aligned}
& \alpha(x)=\alpha(x+y)=\alpha(y), \\
& \beta(x)=\beta(x+y)=\beta(y) .
\end{aligned}
$$

If we select $y=v_{j}$, where $\left\{v_{1}, \ldots, v_{n}\right\}$ is a basis of $\mathbb{R}^{n}$, we obtain

$$
\begin{aligned}
& \alpha\left(v_{j}\right)=\alpha(x)=: \alpha_{0}, \\
& \beta\left(v_{j}\right)=\beta(x)=: \beta_{0} .
\end{aligned}
$$

Hence,

$$
C v_{j}=\alpha_{0} A v_{j}+\beta_{0} B v_{j}, \quad j=1, \ldots, n,
$$

i.e., $C=\alpha_{0} A+\beta_{0} B$, contradicting (I).

Subcase 2(b). We now assume without loss of generality that

$$
\operatorname{dim} \operatorname{span}\{A x, A y, B x\}=3
$$

for some $x, y$, and that

$$
A x \wedge A y \wedge B x \wedge B y=0
$$

for all $x, y$. Let

$$
\Gamma=\{(x, y): A x \wedge A y \wedge B x \neq 0\} .
$$

Again, $\Gamma$ is a Zariski open set, conic in each variable. For $(x, y) \in \Gamma$ we have

$$
B y=\gamma_{1}(x, y) A x+\gamma_{2}(x, y) A y+\gamma_{3}(x, y) B x
$$


for some unique functions $\gamma_{j}$ on $\Gamma$. Since, by assumption, $(\mathrm{H})$ does not hold, again (3) holds and

$$
\begin{aligned}
C(x+y)= & {\left[\alpha(x+y)+\beta(x+y) \gamma_{1}(x, y)\right] A x } \\
& +\left[\alpha(x+y)+\beta(x+y) \gamma_{2}(x, y)\right] A y+\beta(x+y)\left[1+\gamma_{3}(x, y)\right] B x .
\end{aligned}
$$

Moreover,

$$
\begin{aligned}
C(x+y)= & C x+C y \\
=[\alpha(x) & \left.+\beta(y) \gamma_{1}(x, y)\right] A x+\left[\alpha(y)+\beta(y) \gamma_{2}(x, y)\right] A y \\
& +\left[\beta(x)+\beta(y) \gamma_{3}(x, y)\right] B x .
\end{aligned}
$$

Hence,

$$
\left\{\begin{array}{l}
\alpha(x)+\beta(y) \gamma_{1}(x, y)=\alpha(x+y)+\beta(x+y) \gamma_{1}(x, y), \\
\alpha(y)+\beta(y) \gamma_{2}(x, y)=\alpha(x+y)+\beta(x+y) \gamma_{2}(x, y), \\
\beta(x)+\beta(y) \gamma_{3}(x, y)=\beta(x+y)\left(1+\gamma_{3}(x, y)\right) .
\end{array}\right.
$$

If $\beta(x+y)=\beta(y)$ for all $(x, y) \in \Gamma$, then also $\alpha(x+y)=\alpha(y)$ for all $(x, y) \in \Gamma$, and, arguing as in the previous case, we obtain

$$
C=\alpha A+\beta B,
$$

a contradiction.

Assume therefore that $\beta(x+y)-\beta(y) \neq 0$ for some $(x, y) \in \Gamma$, so that generically

$$
\left\{\begin{array}{l}
\gamma_{1}(x, y)=\frac{\alpha(x)-\alpha(x+y)}{\beta(x+y)-\beta(y)}, \\
\gamma_{2}(x, y)=\frac{\alpha(y)-\alpha(x+y)}{\beta(x+y)-\beta(y)}, \\
\gamma_{3}(x, y)=\frac{\beta(x)-\beta(x+y)}{\beta(x+y)-\beta(y)} .
\end{array}\right.
$$

Then equation (6) becomes

$$
\begin{array}{r}
{[\beta(x+y)-\beta(y)] B y=[\alpha(x)-\alpha(x+y)] A x+[\alpha(y)-\alpha(x+y)] A y} \\
+[\beta(x)-\beta(x+y)] B x .
\end{array}
$$

Now we replace $y$ by $t y$, for $t$ so large that also ty lies in the set of generic points, and let $t \rightarrow+\infty$. Since $\alpha$ and $\beta$ are rational functions, homogeneous of degree 0 , we get

$$
\left(\beta^{\prime}(y) \cdot x\right) B y=[\alpha(x)-\alpha(y)] A x-\left(\alpha^{\prime}(y) \cdot x\right) A y+[\beta(x)-\beta(y)] B x .
$$

Since we are assuming that $\beta$ is not constant, it follows that $\beta^{\prime} \neq 0$ and $\beta^{\prime}(y) \cdot x \neq 0$ on a Zariski open set. Hence, generically,

$$
\left\{\begin{array}{l}
\gamma_{1}(x, y)=\frac{\alpha(x)-\alpha(y)}{\beta^{\prime}(y) \cdot x} \\
\gamma_{2}(x, y)=-\frac{\alpha^{\prime}(y) \cdot x}{\beta^{\prime}(y) \cdot x} \\
\gamma_{3}(x, y)=\frac{\beta(x)-\beta(y)}{\beta^{\prime}(y) \cdot x} .
\end{array}\right.
$$


Comparing (8) and (9), we get

$$
\left\{\begin{array}{l}
\frac{\alpha(x+y)-\alpha(x)}{\beta(x+y)-\beta(y)}=\frac{\alpha(y)-\alpha(x)}{\beta^{\prime}(y) \cdot x}, \\
\frac{\alpha(x+y)-\alpha(y)}{\beta(x+y)-\beta(y)}=\frac{\alpha^{\prime}(y) \cdot x}{\beta^{\prime}(y) \cdot x}, \\
\frac{\beta(x+y)-\beta(x)}{\beta(x+y)-\beta(y)}=\frac{\beta(y)-\beta(x)}{\beta^{\prime}(y) \cdot x} .
\end{array}\right.
$$

Proposition 2.1. Let $\alpha, \beta$ be quotients of quadratic forms, with $\beta$ non-constant, satisfying the equations (10). Then there are vectors $u, v, w \in \mathbb{R}^{n}, w \neq 0$, such that $v \wedge w \neq 0$ and

$$
\alpha(x)=\frac{u \cdot x}{w \cdot x}, \quad \beta(x)=\frac{v \cdot x}{w \cdot x} .
$$

Conversely, any pair of functions $\alpha, \beta$ as in (11) satisfies (10).

Proof. Look at the second equation in (10). Replacing $x$ by $t x, t \in \mathbb{R}$, and letting $t \rightarrow+\infty$, we get

$$
\frac{\alpha(x)-\alpha(y)}{\beta(x)-\beta(y)}=\frac{\alpha^{\prime}(y) \cdot x}{\beta^{\prime}(y) \cdot x} .
$$

Similarly, from the third equation in (10), we get

so that

$$
\frac{\beta^{\prime}(x) \cdot y}{\beta(x)-\beta(y)}=\frac{\beta(y)-\beta(x)}{\beta^{\prime}(y) \cdot x}
$$

$$
\left(\beta^{\prime}(x) \cdot y\right)\left(\beta^{\prime}(y) \cdot x\right)=-(\beta(x)-\beta(y))^{2} .
$$

Assume that $\beta=\frac{P}{Q}$, where

$$
P(x)={ }^{t} x D x, \quad Q(x)={ }^{t} x E x,
$$

for symmetric matrices $D$ and $E$. Then

$$
\beta^{\prime}(x) \cdot y=2 \frac{Q(x) P(x, y)-P(x) Q(x, y)}{Q(x)^{2}},
$$

if we set $P(x, y)={ }^{t} x D y$ and $Q(x, y)={ }^{t} x E y$. Also,

$$
\beta(x)-\beta(y)=\frac{P(x) Q(y)-Q(x) P(y)}{Q(x) Q(y)} .
$$

Therefore, (13) implies

$$
\begin{aligned}
& {[P(x) Q(y)-Q(x) P(y)]^{2}} \\
& \quad=-4[Q(x) P(x, y)-P(x) Q(x, y)][Q(y) P(x, y)-P(y) Q(x, y)] .
\end{aligned}
$$

Now the ring $\mathbb{R}\left[x_{1}, \ldots, x_{n}\right]$ is a unique factorization domain (see e.g. [BM]). Therefore, if $P(x) Q(y)-Q(x) P(y)$ were irreducible as a polynomial in $(x, y)$, then we would have, for some $\lambda \in \mathbb{R}$,

$$
Q(x) P(x, y)-P(x) Q(x, y)=\lambda[P(x) Q(y)-Q(x) P(y)],
$$

which is not possible, since the right-hand side is quadratic in $y$, whereas the left-hand side is linear in $y$ and nontrivial. Consequently, there exist polynomials $H_{j}(x, y), j=1,2$, of degree $\geq 1$ such that

$$
P(x) Q(y)-Q(x) P(y)=H_{1}(x, y) H_{2}(x, y) .
$$


Replacing $H_{j}(x, \cdot)$ by its principal part with respect to $y$, we may assume that $H_{j}$ is homogeneous in $y$, and interchanging the roles of $x$ and $y$, we similarly see that we may assume that $H_{j}(x, y)$ is separately homogeneous with respect to $x$ and $y$. Let us assume without loss of generality that $H_{1}(x, y)$ is homogeneous of degree $d \geq 1$ with respect to $y$. Of course, we have $d \leq 2$. If $d=2$, and if $H_{1}=H_{1}(y)$ is a quadratic form in $y$, then $H_{2}=H_{2}(x)$ is a quadratic form in $x$. Interchanging $x$ and $y$ in (15), we then find that $H_{2}(x) H_{1}(y)=-H_{2}(y) H_{1}(x)$, which is not possible. Therefore, $H_{1}$ must indeed be homogeneous of degree 1 in $x$, so that $H_{2}=\xi \cdot x$ for some $\xi \in \mathbb{R}$. Then

$$
Q(y) \frac{P(x)}{\xi \cdot x}-P(y) \frac{Q(x)}{\xi \cdot x}=H_{1}(x, y)
$$

is a polynomial in $x$ for every $y$. But, since $P / Q=\beta$ is not constant, we can find $y, y^{\prime}$ such that $(Q(y), P(y))$ and $\left(Q\left(y^{\prime}\right), P\left(y^{\prime}\right)\right)$ are linearly independent, and therefore also $\frac{P(x)}{\xi \cdot x}$ and $\frac{Q(x)}{\xi \cdot x}$ are polynomials. This shows that there are $v, w \in \mathbb{R}$ such that $P(x)=(v \cdot x)(\xi \cdot x)$ and $Q(x)=(w \cdot x)(\xi \cdot x)$; hence $\beta(x)=\frac{v \cdot x}{w \cdot x}$, as claimed.

By symmetry in $x$ and $y$, we are thus left with the case where $H_{1}$ and $H_{2}$ are both separately homogeneous of degree 1 in $x$ and $y$, i.e., $H_{j}(x, y)={ }^{t} x M_{j} y$ for some matrices $M_{j}$. But then, by (15),

$$
0 \equiv\left({ }^{t} x M_{1} x\right)\left({ }^{t} x M_{2} x\right),
$$

and since $\mathbb{R}\left[x_{1}, \ldots, x_{n}\right]$ is an integral domain, we may assume e.g. that ${ }^{t} x M_{1} x \equiv 0$, so that ${ }^{t} M_{1}=-M_{1}$, and hence $H_{1}(x, y)=-H_{1}(y, x)$. It follows that

$$
H_{1}(x, y)\left[H_{2}(x, y)-H_{2}(y, x)\right] \equiv 0
$$

hence $H_{2}(x, y)=H_{2}(y, x)$, i.e., ${ }^{t} M_{2}=M_{2}$. We have seen that $\omega=H_{1}$ is a skew-symmetric bilinear form, $H_{2}$ is a symmetric bilinear form, and

$$
P(x) Q(y)-Q(x) P(y)=\omega(x, y) H_{2}(x, y) .
$$

But then $\omega$ is irreducible as a polynomial in $(x, y)$, for otherwise we had a factorization $\omega(x, y)=(a \cdot x)(b \cdot y)$, and the skewness of $\omega$ would imply $(a \cdot x)(b \cdot y)=$ $-(b \cdot x)(a \cdot y)$; hence $(a \cdot x) b=-(b \cdot x) a$. This would imply $b=\lambda a$; hence $\omega(x, y)=\lambda(a \cdot x)(a \cdot y)$ would also be symmetric. By (16),$\omega(x, y)$ is thus a prime factor of the right-hand side of (14), so that

$$
Q(x) P(x, y)-P(x) Q(x, y)=q(x) \omega(x, y)
$$

for some quadratic form $q(x)$. Interchanging $x$ and $y$, we also have

$$
Q(y) P(x, y)-P(y) Q(x, y)=-q(y) \omega(x, y) .
$$

If we multiply these two equations and compare with (14), we get

$$
[P(x) Q(y)-Q(x) P(y)]^{2}=4 q(x) q(y) \omega(x, y)^{2} .
$$

This implies that $q \geq 0$ or $q \leq 0$. Assume e.g. that $q \geq 0$. A comparison with (16) then shows that

$$
H_{2}(x, y)^{2}=\left({ }^{t} x M_{2} y\right)^{2}=4 q(x) q(y) .
$$

Choose $y$ such that $q(y) \neq 0$. Then $M_{2} y \neq 0$, and for $x \perp\left(M_{2} y\right)$ we get $0=q(x) q(y)$; hence $q(x)=0$, which means that the hyperplane $\left(M_{2} y\right)^{\perp}$ lies in the radical of the form $q$. This implies that $q$ is of the form $q(x)=(\xi \cdot x)^{2}$ for some $\xi \in \mathbb{R}^{n} \backslash\{0\}$, and hence

$$
H_{2}(x, y)= \pm 2(\xi \cdot x)(\xi \cdot y) .
$$


We thus have $H_{1}(x, y) H_{2}(x, y)=\tilde{H}_{1}(x, y)(\xi \cdot x)$ and have arrived back at the case we had already discussed before.

In conclusion, we have shown that $\beta$ is of the form $\beta(x)=\frac{v \cdot x}{w \cdot x}$. But then one computes that

$$
\begin{gathered}
\beta^{\prime}(x) \cdot y=\frac{(w \cdot x)(v \cdot y)-(v \cdot x)(w \cdot y)}{(w \cdot x)^{2}}, \\
\beta(x)-\beta(y)=\frac{(w \cdot x)(v \cdot y)-(v \cdot x)(w \cdot y)}{(w \cdot x)(w \cdot y)},
\end{gathered}
$$

so that

$$
\frac{\beta(x)-\beta(y)}{\beta^{\prime}(y) \cdot x}=-\frac{w \cdot y}{w \cdot x}
$$

Then, by (12),

$$
\alpha(x)=\alpha(y)-\frac{w \cdot y}{w \cdot x} \alpha^{\prime}(y) \cdot x=\frac{\left(\alpha(y) w-(w \cdot y) \alpha^{\prime}(y)\right) \cdot x}{w \cdot x}=\frac{u \cdot x}{w \cdot x},
$$

as claimed.

Conversely, from (17), (18) and the corresponding formulas for $\alpha$, one easily derives that any pair of functions $\alpha, \beta$ as in (11) satisfies (10).

Lemma 2.2. Let $E$ and $F$ be real $n \times n$ matrices such that $E x=\gamma(x) F x$ for generic $x \in \mathbb{R}^{n}$, where $\gamma(x)=\frac{v \cdot x}{w \cdot x}$ and $v \wedge w \neq 0$. Then there exists a vector $z \in \mathbb{R}^{n}$ such that

$$
E x=(v \cdot x) z, \quad F x=(w \cdot x) z \quad \text { for every } \quad x \in \mathbb{R}^{n} .
$$

Proof. There are no $x, y \in \mathbb{R}^{n}$ such that $(F x) \wedge(F y) \neq 0$, because otherwise the condition would hold for generic $(x, y)$, so that $E(x+y)=\gamma(x+y) F x+\gamma(x+y) F y=$ $\gamma(x) F x+\gamma(y) F y$ would imply $\gamma(x+y)=\gamma(x)=\gamma(y)$ for generic $(x, y)$, so that $\gamma$ would be constant. We may assume that $F \neq 0$. Then $F$ has rank 1 , and so there are nonzero $\eta, z^{\prime} \in \mathbb{R}^{n}$ such that $F x=(\eta \cdot x) z^{\prime}$. Then $E x=\left(\frac{v \cdot x}{w \cdot x} \eta \cdot x\right) z^{\prime}$, so that $x \mapsto \frac{v \cdot x}{w \cdot x} \eta \cdot x$ is a linear mapping. This implies $\eta=\lambda w$, for some real $\lambda$; hence $E x=\lambda(v \cdot x) z^{\prime}$ and $F y=\lambda(w \cdot x) z^{\prime}$. Choosing $z=\lambda z^{\prime}$, the result follows.

To proceed with the discussion of Subcase 2(b), let us now fix $x \in U$ in (6), and let $V_{x}=\operatorname{span}\{A x, B x\}$. Then

$$
B y=\gamma_{2}(x, y) A y \text { modulo } V_{x},
$$

for generic $y$.

Subcase 2b(i). There are some $x \in U$ and $y, z \in \mathbb{R}^{n}$ such that the vectors $A y$ and $A z$ are linearly independent modulo $V_{x}$.

Clearly, the same property then holds for generic $x, y$ and $z$. Arguing as before, we then see that $\gamma_{2}(x, y)$ is constant in $y$ for generic $x$; hence $\gamma_{2}(x, y)=\gamma(x)$. We show that then $\gamma$ must be constant.

By (8) and (11), we have

$$
\gamma(x)=-\frac{((u \cdot x) w-(w \cdot x) u) \cdot y}{((v \cdot x) w-(w \cdot x) v) \cdot y}
$$

for generic $y$. Hence

$$
(\gamma(x) v \cdot x+u \cdot x) w=w \cdot x(u+\gamma(x) v) .
$$


If $\gamma$ were not constant, then we could choose $x, x^{\prime}$ such that $\rho:=\gamma(x) \neq \gamma\left(x^{\prime}\right)=: \rho^{\prime}$ and $w \cdot x \neq 0 \neq w \cdot x^{\prime}$. Then, by (20) $, u+\rho v, u+\rho^{\prime} v \in \mathbb{R} w$, hence $u=\mu w$ and $v=\nu w$ for some $\mu, \nu \in \mathbb{R}$. But then $\alpha(x)=\mu, \beta(x)=\nu$, and hence $C=\mu A+\nu B$, a contradiction.

Let $\rho \in \mathbb{R}$ be such that $\gamma_{2} \equiv \rho$. Then, by (20), $((\rho v+u) \cdot x) w=w \cdot x(u+\rho v)$, which implies that $u+\rho v=\kappa w$ for some $\kappa \in \mathbb{R}$, i.e., $u=\kappa w-\rho v$; hence $\alpha(x)=\kappa-\rho \beta(x)$. Then

$$
(C-\kappa A) x=\beta(x)(B-\rho A) x=\frac{v \cdot x}{w \cdot x}(B-\rho A) x .
$$

Here, because of (I), $v \wedge w \neq 0$ and so, by Lemma 2.2, there is some $z \neq 0$ such that

$$
(C-\kappa A) x=(v \cdot x) z, \quad(B-\rho A) x=(w \cdot x) z .
$$

We show next that there is some $x_{0} \in W:=w^{\perp}$ such that $A x_{0} \notin \mathbb{R} z$.

Otherwise, 21) would imply that $A x, B x \in \mathbb{R} z$ for every $x \in W$. Moreover, $B w \in \operatorname{span}\{A w, z\}$, and hence $A x, B x \in \operatorname{span}\{A w, z\}$ for every $x \in \mathbb{R}^{n}$. This would contradict our assumption that $\operatorname{dim} \operatorname{span}\{A x, A y, B x\}=3$ for some $x, y$. Choose then $x \in W \backslash v^{\perp}$ such that $A x \notin \mathbb{R} z$, and subsequently $\xi$ such that $\xi \cdot(A x)=0$ and $\xi \cdot z \neq 0$. Since $w \cdot x=0$, then also $\xi \cdot(B x)=0$, but $\xi \cdot(C x)=(v \cdot x)(\xi \cdot z) \neq 0$, so that $(\mathrm{H})$ would hold, contradicting our assumption.

Subcase 2b(ii). For every $x \in U$ and $y, z \in \mathbb{R}^{n}$, the vectors $A z$ and $A y$ are linearly dependent modulo $V_{x}$.

In this case, range $A \subset \operatorname{span}\{A x, A y, B x\}$ for every $(x, y) \in \Gamma$. Since, for generic $z$, we have $B z=\gamma_{2}(x, z) A z$ modulo $V_{x}$, then also range $B \subset \operatorname{span}\{A x, A y, B x\}$. Let $V_{x, y}=\operatorname{span}\{A x, A y, B x\},(x, y) \in \Gamma$. Then $V_{x, y}$ does not depend on $(x, y) \in \Gamma$, and we denote this 3 -dimensional space by $V$.

Conclusion of the proof. We have seen that condition $(\mathrm{H})$ holds in Cases 1 to $2 \mathrm{~b}$ (i), and so there only remain Subcase $2 \mathrm{~b}$ (ii) and 2(c). In Subcase $2 \mathrm{~b}$ (ii), the space $V=$ range $A+$ range $B$ is 3 -dimensional, and evidently it is of dimension 2 in Subcase 2(c). Putting $V^{\prime}=$ range ${ }^{t} A+$ range ${ }^{t} B$ and replacing the matrices $A, B$ and $C$ in the discussion of the previous cases by their transposed matrices ${ }^{t} A,{ }^{t} B$ and ${ }^{t} C$, respectively, we may assume in addition that also $\operatorname{dim} V^{\prime} \leq 3$. But, by Proposition 1.4, we then have that $n\left(\mathcal{P}_{\Lambda}\right) \leq \operatorname{dim} V+\operatorname{dim} V^{\prime}$, so that $n\left(\mathcal{P}_{\Lambda}\right) \leq 6$, in contrast to the assumption in Theorem 1.1 .

Remark. It is worth observing that so far we only made use of property (I); the condition $C=[A, B]$ did not play any role (except for Case 1(b), where, however, it was also not really needed, as one easily checks). On the other hand, the proof of Proposition 1.2 will show that the condition $C=[A, B]$ becomes relevant in the lower-dimensional cases.

\section{A ClASS OF EXCEPtional OPERATORS ON $\mathbb{H}_{3}$}

In this section we discuss the lower-dimensional case, that is, $3 \leq n \leq 6$. Our main goal will be to illustrate the complexity of the situation, and we shall therefore restrict ourselves to the simplest case $\mathbb{H}_{3}$.

We are going to consider a class of operators of the form (11) that presents the following features. For some values of the involved parameters, condition (I) implies condition $(\mathrm{H})$; hence the corresponding operators are not locally solvable. 
For the complementary set of values of the parameters, condition (I) does not imply condition $(\mathrm{H})$, and we prove that these operators are in fact locally solvable.

Let

$$
A=\left(\begin{array}{ccc}
0 & 1 & 0 \\
0 & 0 & 1 \\
0 & 0 & 0
\end{array}\right), \quad B=\left(\begin{array}{ccc}
\gamma_{1} & \gamma_{2} & \gamma_{3} \\
\delta_{1} & \delta_{2} & \delta_{3} \\
\varepsilon_{1} & \varepsilon_{2} & \varepsilon_{3}
\end{array}\right)
$$

so that

$$
C=\left(\begin{array}{ccc}
\delta_{1} & \delta_{2}-\gamma_{1} & \delta_{3}-\gamma_{2} \\
\varepsilon_{1} & \varepsilon_{2}-\delta_{1} & \varepsilon_{3}-\delta_{2} \\
0 & -\varepsilon_{1} & -\varepsilon_{2}
\end{array}\right)
$$

Notice that in general we may assume that $A$ (or $B$ ) is in its Jordan canonical form, and we have done so.

Proposition 3.1. Let $\Lambda=A+i B$, where $A$ and $B$ are as above. Let $\mathcal{P}=\mathcal{P}_{\Lambda}$ be defined as in (1). Suppose that condition (I) holds. Then condition $(\mathrm{H})$ is satisfied if and only if one of the following conditions holds:

(i) the parameters $\gamma_{1}, \delta_{1}, \varepsilon_{1}, \varepsilon_{2}, \varepsilon_{3}$ are not all zero; or

(ii) $\gamma_{1}=\delta_{1}=\varepsilon_{1}=\varepsilon_{2}=\varepsilon_{3}=0$, and either $\delta_{2}=0$, or $\delta_{2} \neq 0$ and $\left(\delta_{3}-\gamma_{2}\right)^{2}+$ $4 \gamma_{3} \delta_{2}>0$.

In particular, under these conditions, $\mathcal{P}_{\Lambda}+\mathcal{L}$ is not locally solvable, for every firstorder differential operator $\mathcal{L}$ with smooth coefficients.

Proof. We assume throughout that condition (I) holds.

Observe that, if condition (H) does not hold, then if $y \in \mathbb{R}^{3} \backslash\{0\}$ is such that $A y \wedge B y=0$, then

$$
A y \wedge C y=0 \quad \text { and } \quad B y \wedge C y=0,
$$

and analogously when ${ }^{t} x A \wedge{ }^{t} x B=0$.

Let $y=e_{1}$. Then $A y=0, B y=\left(\gamma_{1}, \delta_{1}, \varepsilon_{1}\right)$ and $C y=\left(\delta_{1}, \varepsilon_{1}, 0\right)$. Then $B y \wedge C y=$ 0 gives $\delta_{1}=\varepsilon_{1}=0$. Moreover, if $x=e_{3}$, then ${ }^{t} x A=0,{ }^{t} x B=\left(0, \varepsilon_{2}, \varepsilon_{3}\right)$ and ${ }^{t} x C=\left(0,0,-\varepsilon_{2}\right)$. Then ${ }^{t} x B \wedge{ }^{t} x C=0$ gives $\varepsilon_{2}=0$. Hence, if $\delta_{1}, \varepsilon_{1}, \varepsilon_{2}$ are not all zero, condition $(\mathrm{H})$ holds.

Suppose then that $\delta_{1}=\varepsilon_{1}=\varepsilon_{2}=0$. Then, condition $(\mathrm{H})$ holds if and only if there exist $x, y \in \mathbb{R}^{3}$ such that

$$
\left\{\begin{array}{l}
x_{1} y_{2}+x_{2} y_{3}=0, \\
x_{1}\left(\gamma_{1} y_{1}+\gamma_{2} y_{2}+\gamma_{3} y_{3}\right)+x_{2}\left(\delta_{2} y_{2}+\delta_{3} y_{3}\right)+\varepsilon_{3} x_{3} y_{3}=0, \\
\left(\delta_{2}-\gamma_{1}\right) x_{1} y_{2}+\left(\delta_{3}-\gamma_{2}\right) x_{1} y_{3}+\left(\varepsilon_{3}-\delta_{2}\right) x_{2} y_{3} \neq 0 .
\end{array}\right.
$$

Observe that, if $\varepsilon_{3} \neq 0$, then the above system can be solved. For, given $y_{2}=$ $\pm y_{3} \neq 0$, select $x_{1}=y_{2}$ and $x_{2}=-y_{3}$. Then choose $y_{3} \neq 0$ so that the third condition above is satisfied (this is possible if $\delta_{3}-\gamma_{2} \neq 0$, by choosing an appropriate sign of $y_{2}$, and if $\delta_{3}-\gamma_{2}=0$, then since $\delta_{2}-\gamma_{1}+\delta_{2}-\varepsilon_{3} \neq 0$, because otherwise $C$ and $A$ would be linearly dependent). Next, choose $x_{3}$ so that the second equation is satisfied. A similar argument shows that if $\gamma_{1} \neq 0$, then $(H)$ holds. This proves (i).

In order to simplify the notation, we relabel the parameters as follows:

$$
\alpha=\gamma_{2}, \beta=\gamma_{3}, \gamma=\delta_{2}, \text { and } \delta=\delta_{3} ;
$$


so that

$$
B=\left(\begin{array}{ccc}
0 & \alpha & \beta \\
0 & \gamma & \delta \\
0 & 0 & 0
\end{array}\right), \quad C=\left(\begin{array}{ccc}
0 & \gamma & \delta-\alpha \\
0 & 0 & -\gamma \\
0 & 0 & 0
\end{array}\right)
$$

Then $(\mathrm{H})$ becomes

$$
\left\{\begin{array}{l}
x_{1} y_{2}+x_{2} y_{3}=0 \\
\alpha x_{1} y_{2}+\beta x_{1} y_{3}+\gamma x_{2} y_{2}+\delta x_{2} y_{3}=0 \\
\gamma x_{1} y_{2}+(\delta-\alpha) x_{1} y_{3}-\gamma x_{2} y_{3} \neq 0 .
\end{array}\right.
$$

If $\gamma=0$, it can be solved (so that (H) holds), since $\delta \neq \alpha$, by condition (I).

Thus, we may assume $\gamma \neq 0$. Notice that we are now reduced to the matrix that gives rise to the operators of the form (2).

Observe that if $y_{3}=0$, then the system (22) has no solution; so assume $y_{3} \neq 0$ and set $t=y_{2} / y_{3}$. Then $x_{2}=-x_{1} t$, and the system (22) reduces to

$$
\left\{\begin{array}{l}
x_{1}\left[-\gamma t^{2}+(\alpha-\delta) t+\beta\right]=0, \\
x_{1}[2 \gamma t+\delta-\alpha] \neq 0
\end{array}\right.
$$

Hence $x_{1} \neq 0$, and the system admits a solution if and only if $(\delta-\alpha)^{2}+4 \beta \gamma>0$, as it is easy to check.

Proposition 3.1 contains in particular the first part of Proposition 1.2 In the last sections, we shall assume that $\gamma \neq 0$ and $(\delta-\alpha)^{2}+4 \beta \gamma \leq 0$, and shall analyze the corresponding class of exceptional operators from Proposition 1.2. We shall show that they admit a tempered fundamental solution; hence they are locally solvable.

\section{A SOLVABILITY CRITERION}

We prove a general criterion for local solvability of an arbitrary left-invariant differential operator $\mathcal{L}$ on the Heisenberg group $\mathbb{H}_{n}$.

For $\mu \in \mathbb{R} \backslash\{0\}$ we consider the Schrödinger representation $\pi_{\mu}$ of $\mathbb{H}_{n}$ on $L^{2}\left(\mathbb{R}^{n}\right)$ :

$$
\left[\pi_{\mu}(x, y, t) \phi\right](v)=e^{i \mu(t+(v+x / 2) y)} \phi(v+x), \quad \phi \in L^{2}\left(\mathbb{R}^{n}\right) .
$$

If $f$ is an integrable function on $\mathbb{H}_{n}$, then the integrated representation is given by

$$
\begin{aligned}
{\left[\pi_{\mu}(f) \phi\right](v) } & =\int_{\mathbb{H}_{n}} f(x, y, t) e^{i \mu(t+(v+x / 2) y)} \phi(v+x) d x d y d t \\
& =\int_{\mathbb{R}^{n}} \overline{\mathcal{F}}_{y, t} f(x, \mu(v+x / 2), \mu) \phi(x+v) d x \\
& =\int_{\mathbb{R}^{n}} \overline{\mathcal{F}}_{y, t} f(u-v, \mu(u+v) / 2, \mu) \phi(u) d u \\
& =: \int_{\mathbb{R}^{n}} \mathcal{K}_{f}^{\mu}(v, u) \phi(u) d u ;
\end{aligned}
$$

here $\overline{\mathcal{F}}_{y, t} f$ denotes the partial Euclidean Fourier co-transform in the indicated variables.

We will also make use of the differential of the Schrödinger representation:

$$
d \pi_{\mu}\left(X_{j}\right)=\partial_{v_{j}}, \quad d \pi_{\mu}\left(Y_{j}\right)=i \mu v_{j}, \quad j=1, \ldots, n ; \quad d \pi_{\mu}(T)=i \mu .
$$


From this and the identity (23) it is easy to check that for any left-invariant differential operator $\mathcal{L}$ we have

$$
\mathcal{K}_{\mathcal{L}^{*} f}^{\mu}=d \pi_{\mu}(\mathcal{L})_{u}^{*} \mathcal{K}_{f}^{\mu}
$$

where $*$ denotes the $L^{2}$-adjoint of an operator.

Theorem 4.1. Let $\mathcal{L}$ be a left-invariant differential operator on the Heisenberg group $\mathbb{H}_{n}$. Suppose that for each $\mu \in \mathbb{R} \backslash\{0\}$ and a.e. $v \in \mathbb{R}^{n}$ there exists a tempered distribution $H_{\mu}(\cdot, v)$ on $\mathbb{R}^{n}$ such that

(i)

$$
d \pi_{\mu}(\mathcal{L}) H_{\mu}(\cdot, v)=\delta(v-\cdot) ;
$$

(ii) for every function $\varphi \in \mathcal{D}\left(\mathbb{R}^{n} \times \mathbb{R}^{n}\right)$, the mapping $v \mapsto\left\langle H_{\mu}(\cdot, v), \varphi(\cdot, v)\right\rangle$ is integrable and defines a tempered distribution

$$
\left\langle H_{\mu}, \varphi\right\rangle:=\int_{\mathbb{R}^{n}}\left\langle H_{\mu}(\cdot, v), \varphi(\cdot, v)\right\rangle d v ;
$$

(iii) if we set $\tilde{H}_{\mu}(x, \eta)=H_{\mu}\left(\frac{\eta}{\mu}+\frac{x}{2}, \frac{\eta}{\mu}-\frac{x}{2}\right)$, where the change of variables is to be understood in the sense of distributions, then

$$
\langle\tilde{H}, \psi\rangle:=\int_{\mathbb{R} \backslash\{0\}}\left\langle\tilde{H}_{\mu}, \psi(\cdot, \mu)\right\rangle d \mu
$$

defines a tempered distribution $\tilde{H}$, in a similar way as described in (ii), i.e., $\tilde{H} \in \mathcal{S}^{\prime}\left(\mathbb{R}^{n} \times \mathbb{R}^{n} \times \mathbb{R}\right)$.

Then $\mathcal{L}$ admits a tempered fundamental solution.

Proof. By assumption, there exists a tempered distribution $K$ on $\mathbb{H}_{n}$ such that $\overline{\mathcal{F}}_{y, t} K=\tilde{H}$.

Then, for $f \in \mathcal{S}\left(\mathbb{H}_{n}\right)$ we have

$$
\begin{aligned}
& (\mathcal{L} K, f)=\left(K, \mathcal{L}^{*} f\right)=c_{n}\left(\overline{\mathcal{F}}_{y, t} K, \overline{\mathcal{F}}_{y, t}\left(\mathcal{L}^{*} f\right)\right)
\end{aligned}
$$

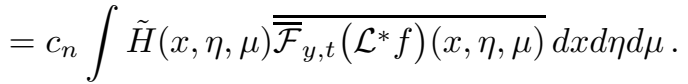

Next, we perform the change of variables

$$
\left\{\begin{array} { l } 
{ x = u - v , } \\
{ \eta = \mu ( \frac { u + v } { 2 } ) , }
\end{array} \quad \text { i.e., } \quad \left\{\begin{array}{l}
u=\frac{\eta}{\mu}+\frac{x}{2}, \\
v=\frac{\eta}{\mu}-\frac{x}{2},
\end{array}\right.\right.
$$

and use (24). Then the right-hand side in (25) equals an absolute constant times

$$
\begin{aligned}
& \int \tilde{H}_{\mu}(u-v, \mu(u+v) / 2) \overline{\mathcal{K}_{\mathcal{L}^{*} f}^{\mu}(v, u)} d u d v\left|\frac{\mu}{2}\right|^{n} d \mu \\
& =\int H_{\mu}(u, v) \overline{d \pi_{\mu}(\mathcal{L})_{u}^{*} \mathcal{K}_{f}^{\mu}(v, u)} d u d v\left|\frac{\mu}{2}\right|^{n} d \mu \\
& =\int d \pi_{\mu}(\mathcal{L})_{u} H_{\mu}(u, v) \overline{\left(\overline{\mathcal{F}}_{y, t} f\right)(u-v, \mu(u+v) / 2, \mu)} d u d v\left|\frac{\mu}{2}\right|^{n} d \mu \\
& =\int \overline{\mathcal{F}_{y, t} f(0, \mu v, \mu)} d v\left|\frac{\mu}{2}\right|^{n} d \mu \\
& =c \overline{f(0,0,0)}
\end{aligned}
$$


for some absolute constant $c$. Of course, some of the integrals in this argument have to be interpreted in the distributional sense. This shows that $K$ is a multiple of a fundamental solution for $\mathcal{L}$.

\section{Solvabilty of the EXCEPtional operators}

We wish to prove the solvability of the operator $\mathcal{P}$ in (2). By making the symplectic change of variables $\left(x_{j}, y_{j}\right) \mapsto\left(-y_{j}, x_{j}\right), j=1,2$ (where $x_{3}, y_{3}$ remain unchanged), this is equivalent to proving the local solvability of the operator

$$
\tilde{\mathcal{P}}=(1+i \alpha) Y_{1} X_{2}+i \beta Y_{1} Y_{3}+i \gamma Y_{2} X_{2}+(1+i \delta) Y_{2} Y_{3} .
$$

Recall that we assume that $\gamma \neq 0$ and

$$
(\delta-\alpha)^{2}+4 \beta \gamma \leq 0
$$

if we set $\varrho:=\beta \gamma-\alpha \delta+1$ and $c:=\alpha+\delta$, then the condition (27) is equivalent to

$$
\varrho \leq 1-\frac{c^{2}}{4} \text {. }
$$

Notice that we may assume that $\gamma>0$, and we do so in what follows.

By applying the Schrödinger representation, the operator $\tilde{\mathcal{P}}$ is transformed into $d \pi_{\mu}(\tilde{\mathcal{P}})=: i \mu D$, where

$$
D=\left[(1+i \alpha) x_{1}+i \gamma x_{2}\right] \partial_{x_{2}}+\left[\beta x_{1}+(\delta-i) x_{2}\right]\left(-\mu x_{3}\right) .
$$

Notice that for $x_{1} \in \mathbb{R} \backslash\{0\}$, the ratio $\left(x_{1}+i y_{1}\right) /\left(x_{1}+i y_{2}\right)$ takes values in either the lower or the upper half-plane, according to the sign of $x_{1}\left(y_{1}-y_{2}\right)$.

For $x_{1} \neq 0$ and for $x_{2} \geq s$ (or for $x_{2} \leq s$ ) we define

$$
h(x, s)=e^{\frac{1+i \delta}{\gamma} x_{3}\left(x_{2}-s\right)}\left[\frac{x_{1}+i\left(\alpha x_{1}+\gamma x_{2}\right)}{x_{1}+i\left(\alpha x_{1}+\gamma s\right)}\right]^{\kappa},
$$

where

$$
\kappa=\kappa_{1}+i \kappa_{2}:=\frac{x_{1} x_{3}}{\gamma^{2}}[-(\alpha+\delta)+i \varrho],
$$

and where we select the branch of the $\operatorname{logarithm} \log \zeta$ that equals 0 when $\zeta=1$.

We wish to show that $h$ is bounded when $x_{3}\left(s-x_{2}\right) \geq 0$.

Lemma 5.1. For $t \in \mathbb{R}$ set

$$
J(t)=t-\int_{0}^{|t|} \frac{c s}{1+s^{2}} d s-\int_{0}^{t} \frac{\varrho}{1+s^{2}} d s .
$$

Assume that (28) holds. Then the function $J(t)$ is increasing for $t \in \mathbb{R}$.

Proof. We have that

$$
J^{\prime}(t)=1-\frac{c t}{1+t^{2}}-\frac{\varrho}{1+t^{2}}=1-\frac{c t+\varrho}{1+t^{2}},
$$

and the claim follows immediately from (28).

Lemma 5.2. Let $h$ be defined as in (30). Then

$$
\left|h\left(x_{1}, x_{2}, x_{3}, s\right)\right| \leq 1
$$

when $x_{3}\left(s-x_{2}\right) \geq 0$. 
Proof. Notice that

$$
\begin{aligned}
\left|h\left(x_{1}, x_{2}, x_{3}, s\right)\right| & =e^{\frac{x_{3}\left(x_{2}-s\right)}{\gamma}}\left|\left[\frac{1+i\left(\alpha+\gamma \frac{x_{2}}{x_{1}}\right)}{1+i\left(\alpha+\gamma \frac{s}{x_{1}}\right)}\right]^{\kappa_{1}+i \kappa_{2}}\right| \\
& =e^{\frac{x_{3}\left(x_{2}-s\right)}{\gamma}}\left[\frac{1+\left(\alpha+\gamma \frac{x_{2}}{x_{1}}\right)^{2}}{1+\left(\alpha+\gamma \frac{s}{x_{1}}\right)^{2}}\right]^{\frac{\kappa_{1}}{2}} e^{-\kappa_{2}\left(\arctan \left(\alpha+\gamma \frac{x_{2}}{x_{1}}\right)-\arctan \left(\alpha+\gamma \frac{s}{x_{1}}\right)\right) .}
\end{aligned}
$$

Put

$$
b:=\alpha+\gamma \frac{x_{2}}{x_{1}}, \quad a:=\alpha+\gamma \frac{s}{x_{1}}
$$

so that

$$
\begin{aligned}
\log \mid & h\left(x_{1}, x_{2}, x_{3}, s\right) \mid \\
= & \frac{x_{1} x_{3}}{\gamma^{2}}\left[(b-a)-\frac{\alpha+\delta}{2} \log \left(\frac{1+b^{2}}{1+a^{2}}\right)-\varrho(\arctan b-\arctan a)\right] \\
= & \frac{x_{1} x_{3}}{\gamma^{2}}\left[(b-a)-\int_{|a|}^{|b|} \frac{(\alpha+\delta) t}{1+t^{2}} d t-\int_{a}^{b} \frac{\varrho}{1+t^{2}} d t\right] \\
= & \frac{x_{1} x_{3}}{\gamma^{2}}[J(b)-J(a)] .
\end{aligned}
$$

We wish to show that $\log \left|h\left(x_{1}, x_{2}, x_{3}, s\right)\right| \leq 0$ for $x_{3}\left(s-x_{2}\right) \geq 0$. This follows at once from (34), (33) and Lemma 5.1

We now define the solution kernel for the differential operator $d \pi_{\mu}(\tilde{\mathcal{P}})=: i \mu D$. For $\mu \neq 0$ and $x \in \mathbb{R}^{3}, s \in \mathbb{R}$ we set

$$
G_{\mu}(x ; s)= \begin{cases}\frac{1}{i \mu} \frac{h\left(x_{1}, x_{2},-\mu x_{3}, s\right)}{(1+i \alpha) x_{1}+i \gamma s} \chi_{\left\{s<x_{2}\right\}} & \text { if }-\mu x_{3}<0, \\ -\frac{1}{i \mu} \frac{h\left(x_{1}, x_{2},-\mu x_{3}, s\right)}{(1+i \alpha) x_{1}+i \gamma s} \chi_{\left\{x_{2}<s\right\}} & \text { if }-\mu x_{3}>0 .\end{cases}
$$

Notice that $\left|h\left(x_{1}, x_{2},-\mu x_{3}, s\right)\right| \leq 1$ in (35), by Lemma 5.2 Next, for $f \in \mathcal{S}\left(\mathbb{R}^{3}\right)$ we set

$$
\varphi(x)=\int_{-\infty}^{+\infty} G_{\mu}(x ; s) f\left(x_{1}, s, x_{3}\right) d s .
$$

Lemma 5.3. Let $f \in \mathcal{S}\left(\mathbb{R}^{3}\right)$ be a given Schwartz function. Let $\varphi$ be defined as above. Then $u$ satisfies the differential equation $d \pi_{\mu}(\tilde{\mathcal{P}}) \varphi=f$.

Proof. In order to study $d \pi_{\mu}(\tilde{\mathcal{P}})$ we introduce the differential operator

$$
L=a\left(x_{2}\right) \partial_{x_{2}}-b\left(x_{2}\right),
$$

where $a$ is a non-vanishing function. It is easy to check that for a given $g \in \mathcal{S}(\mathbb{R})$ the equation $L \psi=g$ admits the solution

$$
\psi\left(x_{2}\right)=\int_{s_{0}}^{x_{2}} \frac{g(s)}{a(s)} e^{\int_{s}^{x_{2}} \frac{b(t)}{a(t)} d t} d s .
$$

Now we set, for $x_{1}, x_{3}$ and $\mu$ fixed,

$$
\begin{aligned}
& a\left(x_{2}\right)=(1+i \alpha) x_{1}+i \gamma x_{2}, \\
& b\left(x_{2}\right)=\left((i-\delta) x_{2}-\beta x_{1}\right)\left(-\mu x_{3}\right),
\end{aligned}
$$


so that

$$
\begin{aligned}
\int_{s}^{x_{2}} \frac{b(t)}{a(t)} d t & =\left(-\mu x_{3}\right) \int_{s}^{x_{2}} \frac{(i-\delta) t-\beta x_{1}}{(1+i \alpha) x_{1}+i \gamma t} d t \\
& =\frac{1+i \delta}{\gamma}\left(-\mu x_{3}\right) \int_{s}^{x_{2}}\left(1-\frac{\left(-\frac{\beta \gamma}{1+i \delta}+1+i \alpha\right) x_{1}}{(1+i \alpha) x_{1}+i \gamma t}\right) d t \\
& =\frac{1+i \delta}{\gamma}\left(-\mu x_{3}\right)\left(x_{2}-s\right) \\
& -\left[\beta+\frac{(1+i \delta)(1+i \alpha)}{\gamma}\right] \frac{x_{1}\left(-\mu x_{3}\right)}{i \gamma} \log \left[\frac{x_{1}+i\left(\alpha x_{1}+\gamma x_{2}\right)}{x_{1}+i\left(\alpha x_{1}+\gamma s\right)}\right]
\end{aligned}
$$

Then,

$$
\begin{aligned}
e^{\int_{s}^{x_{2}} \frac{b(t)}{a(t)} d t} & =e^{\frac{1+i \delta}{\gamma}\left(-\mu x_{3}\right)\left(x_{2}-s\right)}\left[\frac{x_{1}+i\left(\alpha x_{1}+\gamma x_{2}\right)}{x_{1}+i\left(\alpha x_{1}+\gamma s\right)}\right]^{[i \beta \gamma+(1+i \delta)(-\alpha+i)] \frac{x_{1}\left(-\mu x_{3}\right)}{\gamma^{2}}} \\
& =e^{\frac{1+i \delta}{\gamma}\left(-\mu x_{3}\right)\left(x_{2}-s\right)}\left[\frac{x_{1}+i\left(\alpha x_{1}+\gamma x_{2}\right)}{x_{1}+i\left(\alpha x_{1}+\gamma s\right)}\right]^{\frac{x_{1}\left(-\mu x_{3}\right)}{\gamma^{2}}[-(\alpha+\delta)+i \varrho]} \\
& =h\left(x_{1}, x_{2},-\mu x_{3}, s\right) .
\end{aligned}
$$

Replacing $\psi$ by $\varphi$ and $g$ by $f$ in (37), inserting the expression of $a$ and choosing either $s_{0}=-\infty$ or $s_{0}=\infty$, we obtain the desired conclusion.

Let us define

$$
H_{\mu}(u, v)=\int G_{\mu}(u ; s) \delta_{v}\left(u_{1}, s, u_{3}\right) d s=G_{\mu}\left(u ; v_{2}\right) \delta\left(v_{1}-u_{1}\right) \delta\left(v_{3}-u_{3}\right) .
$$

Proposition 5.4. The distributions $H_{\mu}$ satisfy the conditions of Theorem 4.1. Consequently, the operator $\mathcal{P}=\mathcal{P}_{\Lambda}$ in (2) with $\alpha, \beta, \gamma, \delta \in \mathbb{R}, \gamma \neq 0$ and $(\delta-\alpha)^{2}+$ $4 \beta \gamma \leq 0$ admits a tempered fundamental solution, and hence is locally solvable.

Proof. Clearly, for every $\mu \neq 0$ and a.e. $v \in \mathbb{R}^{3}, H_{\mu}(\cdot, v)$ is a bounded measure, hence a tempered distribution. Moreover, it follows from Lemma 5.3 that $d \pi_{\mu}(\tilde{\mathcal{P}}) H_{\mu}(\cdot, v)=\delta(v-\cdot)$, so that condition (i) in Theorem 4.1 holds true.

Next, for $\varphi \in \mathcal{D}\left(\mathbb{R}^{3} \times \mathbb{R}^{3}\right)$ we have

$$
\left\langle H_{\mu}(\cdot, v), \varphi(\cdot, v)\right\rangle=\int G_{\mu}\left(\left(v_{1}, u_{2}, v_{3}\right) ; v_{2}\right) \varphi\left(\left(v_{1}, u_{2}, v_{3}\right), v\right) d u_{2} .
$$

Hence

$$
\left|\left\langle H_{\mu}(\cdot, v), \varphi(\cdot, v)\right\rangle\right| \leq|\mu|^{-1}\left|\left(v_{1}, \alpha v_{1}+\gamma v_{2}\right)\right|^{-1} \int\left|\varphi\left(\left(v_{1}, s, v_{3}\right), v\right)\right| d s .
$$

Since $\gamma \neq 0$, this implies that $H_{\mu}$ also satisfies condition (ii) in Theorem 4.1.

Finally, we have

$$
\tilde{H}(x, \eta, \mu)=G_{\mu}\left(\frac{\eta}{\mu}+\frac{x}{2} ; \frac{\eta_{2}}{\mu}-\frac{x_{2}}{2}\right) \delta\left(x_{1}\right) \delta\left(x_{3}\right) .
$$

Thus

$$
\tilde{H}(x, \eta, \mu)=\frac{\tilde{h}(x, \eta, \mu)}{(1+i \alpha) \eta_{1}+i \gamma\left(\eta_{2}-\frac{\mu x_{2}}{2}\right)} \delta\left(x_{1}\right) \delta\left(x_{3}\right),
$$

where $|\tilde{h}| \leq 1$. This shows immediately that $\tilde{H} \in \mathcal{S}^{\prime}\left(\mathbb{R}^{3} \times \mathbb{R}^{3} \times \mathbb{R}\right)$, and thus also (iii) is satisfied. 


\section{REFERENCES}

[BF] R. Beals and C. Fefferman, On local solvability of linear partial differential equations, Ann. Math. 97 (1973), 482-498. MR 50:5233

[BGH] L. Boutet de Monvel, A. Grigis, and G. Helffer, Parametrixes d'opérateurs pseudodifférentiels à caractéristiques multiples, Astérisque 34-35 (1976), 93-121. MR 58:12046

[BM] G. Birkhoff and S. MacLane, Algebra, The MacMillan Company, London, 1967. MR 35:5266

[CR] L. Corwin and L. P. Rothschild, Necessary conditions for local solvability of homogeneous left invariant differential operators on nilpotent Lie groups, Acta Math. 147 (1981), 265288. MR 83b:22010

[F] G. B. Folland, Harmonic analysis on phase space, Annals of Math. Studies 122, Princeton Univ. Press, Princeton, 1989. MR 92k:22017

[G] V. V. Grušin, Extension of smoothness to solutions of differential equations of smooth type, Soviet Math. Doklaky 4 (1963), 248-252. MR 26:2717

$[\mathrm{HeN}]$ B. Helffer and J. Nourrigat, Caractérisation des opérateurs hypoelliptiques homogènes invariants à gauche sur un groupe nilpotent gradué, Comm. in Partial Differential Equations 4 (1979), 899-958. MR 81i:35034

[H1] L. Hörmander, Differential operators of principal type, Math. Ann. 140 (1960), 124-146. MR 24:A434

[H2] Differential equations without solutions, Math. Ann. 140 (1960), 169-173. MR 26:5279

[H3] A class of hypoelliptic pseudodifferential operators with double characteristics, Math. Ann. 217 (1975), 165-188. MR 51:13774

[KM] G. E. Karadzhov and D. Müller, A remarkable class of second-order differential operators on the Heisenberg group $\mathbb{H}_{2}$, Math. Ann. 320 (2001), 731-755. MR 2002g:35001

[L-B] P. Lévy-Bruhl, Résolubilité locale et globale d'opérateurs invariants du second ordre sur des groupes de Lie nilpotents Bull. Sci. Math. 104 (1980), 369-391. MR 82d:58067

[L] H. Lewy, An example of a smooth differential operator without solution, Ann. Math. 66 (1957), 155-158. MR 19:551d

[MPR] D. Müller, M. M. Peloso, and F. Ricci, On local solvability for complex coefficient differential operators on the Heisenberg group, J. reine angew. Math. 513 (1999), 181-234. MR 2000h:35003

[MR1] D. Müller and F. Ricci, Analysis of second-order differential operators on the Heisenberg group II, J. Funct. Anal. 108 (1992), 296-346. MR 93j:22019

[MR2] Solvability for a class of doubly characteristic differential operators on 2-step nilpotent groups, Ann. Math. 143 (1996), 1-49. MR 96m:22018

[MR3] Solvability of second-order left-invariant differential operators on the Heisenberg group satisfying a cone condition, J. Analyse Math., to appear.

[MZ] D. Müller and Z. Zhang, A class of solvable non-homogeneous differential operators on the Heisenberg group, Studia Math. 148 (2001), 87-96. MR 2002m:35031

[NT] L. Nirenberg and F. Treves, On local solvability of linear partial differential equations: I, II Comm. Pure Appl. Math., 23 (1970), 1-38, 459-509 and 24 (1971), 279-288. MR 41:9064a MR 41:9064b MR 55:8599

[R] L. P. Rothschild, Local solvability of second-order differential operators on nilpotent Lie groups, Ark. för Mat. 19 (1981), 145-175. MR 84e:58080

[S] J. Sjöstrand, Parametrices for pseudodifferential operators with multiple characteristics, Ark. för Mat. 12 (1974), 85-130. MR 50:5236

[T] F. Treves, Introduction to Pseudodifferential and Fourier Integral Operators, Vols. 1, 2, Plenum Press, New York, 1980. MR 82i:35173 MR 82i:58068

Mathematisches Seminar, C.A.-Universität Kiel, Ludewig-Meyn-Strasse 4, D-24098 Kiel, Germany

E-mail address: mueller@math.uni-kiel.de

Dipartimento di Matematica, Corso Duca degli Abruzzi 24, Politecnico di Torino, 10129 TORINO, ITALY

E-mail address: peloso@calvino.polito.it 